\title{
Antimyeloperoxidase antibodies in individuals with occupational exposure to silica
}

Ingeborg Wichmann, Julio Sanchez-Roman, Javier Morales, Maria Jesús Castillo, Celia Ocaña, Antonio Nuñez-Roldan

\begin{abstract}
Objective-To determine the prevalence of autoantibodies to myeloperoxidase (MPO) in a series of patients exposed to silica.

Methods-The study included 52 patients with occupational exposure to silica (mean exposure time seven years) and a control group comprising seven patients with progressive systemic sclerosis (PSS), six patients with systemic lupus erythematosus (SLE), and 15 healthy individuals. Antibodies to MPO were detected using commercial enzyme linked immunosorbent assay (ELISA) plates coated with MPO. Indirect immunofluorescence studies for antineutrophil cytoplasmic antibodies were performed using ethanol and formol fixed neutrophils. Clinical and biological data of individuals exposed to silica were recorded (published previously).

Results-Antibodies to MPO were detected in 14 individuals exposed to silica (27\%). There was a statistically significant difference in anti-MPO ELISA units between the healthy subjects and patients (SLE, PSS, silica exposed individuals) $(p<0.01)$, but no difference between the different disease groups.

Conclusions-Individuals chronically exposed to silica, whether or not they have a connective tissue disease, have levels of antibodies to MPO (as detected by ELISA) that are greater than those found in the normal population, but similar to those in patients with systemic diseases not induced by silica (SLE/PSS).
\end{abstract}

Servicio de Inmunología and Unidad de

Colagenosis, Hospital Universitario

Virgen del Rocío, Servicio Andaluz de Salud,

Sevilla, Spain

I Wichmann

J Sanchez-Roman

J Morales

M J Castillo

C Ocaña

A Nuñez-Roldan

Correspondence to

Antonio Nuñez-Roldan Servicio de Inmunologia, Hospital Universitario Virgen del Rocio

Avenida Manuel Siurot s/n 41013 Sevilla, Spain

Accepted for publication 30 October 1995
(Ann Rheum Dis 1996; 55: 205-207)

Evidence that occupational exposure to silica, which has long been known to be associated with lung fibrosis, is also associated with autoimmunity, was reinforced by the observation that workers from a factory that produced scouring powder with an increased silica content exhibited a high prevalence of clinical and biological autoimmune manifestations. ${ }^{12}$ Among a group of 50 workers, symptoms of systemic disease were evident in $32(64 \%)$, and antinuclear antibodies were present in 36 $(72 \%)$. There have also been reports of antibodies to MPO in eight silicotic patients with renal involvement. ${ }^{34}$

Until recently, ANCAs were reported only in relation to vasculitic syndromes. However, the disease spectrum with which this family of autoantibodies is associated has broadened, and they have now been described in diseases such as rheumatoid arthritis, ${ }^{5}$ ulcerative colitis, ${ }^{6}$ cholangitis, ${ }^{7}$ and idiopathic and drug induced systemic lupus erythematosus (SLE). ${ }^{8}$

In the present study, we investigated the presence of antineutrophil cytoplasmic antibodies (ANCAs) with antimyeloperoxidase (MPO) specificity in 52 workers from the factory referred to above.

\section{Patients and methods}

The study included 52 subjects (table) with a history of occupational exposure to silica at a scouring powder factory (mean exposure time seven years). Thirty eight exhibited features of systemic illness (15 of them undefined). Twenty two patients developed a defined systemic disease: 16 SLE and 12 progressive systemic sclerosis (PSS); six presented SLE + PSS concurrently. Fifteen patients showed a sicca syndrome, in three associated with features of undefined systemic disease and in the remainder associated with a defined connective tissue disease (four SLE, two PSS, and six SLE + PSS). Two patients had glomerulonephritis (one of them with SLE, and the other without any associated systemic disease). The remaining subjects had no renal involvement. None of these patients exposed to silica had a vasculitic syndrome. Fifteen healthy subjects, in addition to seven patients with PSS and six patients with SLE, all of whom had not been exposed to silica, were included in the study as controls.

Quantification of IgG MPO antibodies was performed with a commercial ELISA (Bios $\mathrm{GmbH}$, Germany). The assay was tested and

Clinical diagnosis in 52 subjects exposed to silica and 28 controls

\begin{tabular}{lcc}
\hline & No & $\%$ \\
\hline Silica group & 52 & 100 \\
Systemic lupus erythematosus (SLE) & 16 & $30 \cdot 7$ \\
Systemic sclerosis (SSc) & 12 & 23 \\
Polymyositis (PM) & 1 & $1 \cdot 9$ \\
Undefined collagen disease & 15 & $28 \cdot 8$ \\
Overlap syndromes $^{\star}$ & 7 & $13 \cdot 5$ \\
SLE-SSc & 6 & $11 \cdot 5$ \\
PM-SSc & 1 & $1 \cdot 9$ \\
Asymptomatic subjects & 15 & $28 \cdot 8$ \\
Control group & 28 & 100 \\
Healthy & 15 & $53 \cdot 6$ \\
Systemic lupus erythematosus & 6 & $21 \cdot 4$ \\
Systemic sclerosis & 7 & 25 \\
\hline
\end{tabular}

*Included in the four first clinical diagnoses. 
provided at the 1994 Workshop on Autoimmunity held by the Spanish Society of Immunology; sera were diluted as recommended by the manufacturer.

Sera were further studied by indirect immunofluorescence on ethanol and formol fixed neutrophils for ANCA detection (INOVA Diagnostics Inc, San Diego, California), and on rat (liver, kidney, stomach) and human (epithelial 2) substrates for antinuclear antibodies (Biosystems S A Barcelona, Spain).

ANALYSIS OF RESULTS

Evaluation of results was performed with the standard curve obtained from control sera included in the commercial ELISA. The cut off level (13 ELISA units) was set by calculating the mean of values from healthy people $(6 \cdot 83$ ELISA units) plus 2 standard deviations $(\mathrm{SD}=2 \cdot 88)$.

Data were analysed statistically by the nonparametric Kruskal-Wallis test and by the Tukey multiple comparison test.

\section{Results}

Among the subjects exposed to silica, 14 had antibodies against MPO (27\%). Prevalence of anti-MPO in groups not exposed to silica (SLE, PSS, and healthy individuals) was respectively $0(0 \%), 5(71 \%)$, and $0(0 \%)$. There was a statistically significant difference in the absorbance values when the Kruskall-Wallis test was applied $(p<0.01)$ between the healthy group (6.83 (SD 2.88) ELISA units), patients with SLE (11.16 (1.03) ELISA units), those with PSS $(21.07$ (16.42) ELISA units), and individuals exposed to silica $(14.07(9.66)$ ELISA units)(figure).

Using Tukey's multiple comparison test, no difference was found between the patients with SLE and those with PSS, and there was no difference between individuals with or without systemic disease who had been exposed to silica compared with the disease control groups (idiopathic SLE or PSS); however, the test discriminated between the healthy group and the three disease groups $(p<0.01)$.

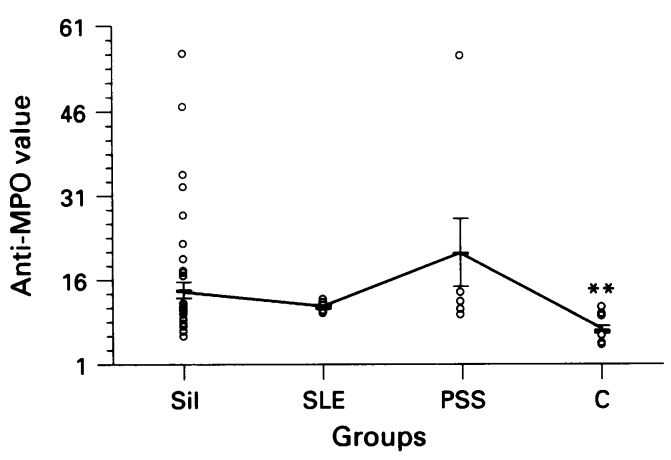

Levels of myeloperoxidase antibodies (Anti-MPO) as measured by ELISA in 52 individuals exposed to silica (Sil), six patients with systemic lupus erythematosus (SLE), seven patients with progressive systemic sclerosis (PSS), and 15 healthy individuals (controls (C)). Mean (SEM) are shown. ${ }^{\star x_{p}}<0.01$ (Kruskall-Wallis test) compared with patient groups.
There were no significant differences between those individuals exposed to silica who did or did not have collagen disease with regard to their levels of MPO antibodies. One of the two patients with renal involvement had a significant titre of MPO antibodies, but the serum from the other was negative (serum dated 1985-the beginning of dialysis-and serum from 1994).

The indirect immunofluorescence test performed simultaneously on ethanol and formol fixed neutrophils gave negative results for the sera of all patients exposed to silica, except for the sample from the patient with renal disease, which showed a p-ANCA pattern at $1 / 32$ dilution and was positive by ELISA (28 ELISA units). The presence of MPO antibodies did not correlate with the presence of antinuclear antibodies.

\section{Discussion}

Antineutrophil cytoplasmic antibodies are a heterogeneous group of autoantibodies directed against cytoplasmic components of neutrophils and monocytes. By means of different fixation procedures (ethanol and formol), two major patterns of indirect immunofluorescence have been described: the cytoplasmic or c-ANCA pattern, generally well correlated with PR3 antibodies, and the peripheral or p-ANCA pattern, associated mainly with MPO antibodies, but also with antibodies to lactoferrin, cathepsin G, elastase, and lysozyme. In addition, some ANCAs have been described as having an atypical pattern (aANCA); the specificity of their targets remains unknown.

The discovery of autoantibodies to ANCAs and their association with different types of systemic vasculitis has proved very important for the diagnosis and follow up of the patients. ${ }^{9}$ More recently, ANCAs have been detected in different rheumatic, bowel, and other diseases, and have also been reported in eight silicotic patients with renal involvement.

We previously reported our experience with 50 individuals with occupational exposure to silica during their work for several years at a scouring powder factory. ${ }^{1}$ Thirty two of them showed many clinical and immunobiological features of autoimmune disease. Antinuclear antibodies were present in $72 \%$ of these individuals. At the time of the study they had no abnormalities of urine sediment, no proteinuria, no haematuria, and a normal creatinine clearance. These clinical data have not changed during the past four years.

To establish the anti-MPO status of these workers who had extensive exposure to silica, we studied their sera by means of an ELISA, using purified MPO antigen (Bios, Germany), and including some new cases. Among 52 patients studied, 14 were positive (cut off $=13$ ELISA units). One patient suffering chronic renal insufficiency (extracapillary glomerulopathy), who was not included in our first series but who worked at the same factory, showed no evidence of MPO antibodies in a serum from 1985 or in a serum collected during the 
present study. Another patient was positive by indirect immunofluorescence and ELISA and had renal involvement. Two other patients who worked at the same factory had renal disease (extracapillary glomerulonephritis), but they died before our study began and we do not know their anti-MPO status. Because only two patients had renal disease, and just one of them was positive for anti-MPO, we are unable to evaluate the association of anti-MPO with nephropathy that has been described by others. $^{34}$

One of the surprising results of this study was the absence of p-ANCA positivity by indirect immunofluorescence in all individuals exposed to silica except one patient with renal involvement. A similar paradox has been observed in some cases of necrotising and crescentic glomerulonephritis and SLE. ${ }^{10}$ One explanation of this finding could be that these antibodies are recognising a hidden antigen; alternatively, they may be destroyed by the procedures of cell fixation in indirect immunofluorescence, but exposed during ELISA.

In conclusion, our study demonstrates that, in addition to the common occurrence of antinuclear antibodies among individuals chronically exposed to silica, ${ }^{1}$ these patients have a high prevalence of MPO ANCA antibodies as detected by ELISA. Our findings also suggest that there is a polyclonal immune activation elicited as a result of chronic contact with the agent.
We wish to thank Dr Berta Sanchez for helpful discussion. This study was supported by grants from Dirección General de Investigación Científica y Técnica (DGICYT, PB92/1107), from Fondo de Investigaciones Sanitarias, Ministerio de fromidad y Consumo, Spain (FIS 93/0355 and FIS 94/0803), and from Plan Andaluz de Investigación (PAI, grupo 3151), Consejeria de Educación y Ciencia, Junta de Andalucia.

1 Sanchez-Roman J, Wichmann I, Salaberri J, Varela J M, Nuñez-Roldan A. Multiple clinical and biological autoimmune manifestation in 50 workers after occupational

2 Nuñez-Roldan A, Sanchez-Roman J, Wichmann I. Silicon nephropathy and anti-myeloperoxidase antibodies. Ann Rheum Dis 1994; 53: 781-2.

3 Chevailler A, Subra J F, Renier G, et al. Antimyeloperoxidase antibodies and silicosis with renal involvement: new association or coincidental event. $\mathrm{Am}$ f Kidney Dis 1991; 28: 213 (Abstract 61).

4 Chevailler A, Subra J F, Carrère F, et al. Silicon nephropathy and anti-myeloperoxidase antibodies. Ann Rheum Dis 1994; 53: 781-2.

5 Savige J A, Gallicchio M C, Stockman A, et al. Antineutrophil cytoplasm antibodies in rheumatoid arthritis. Clin Exp Immunol 1991; 86: 92-8.

6 Saxon A, Shanahan F, Landers C, Ganz T, Targan S. A distinct subset of anti-neutrophil cytoplasmic antibodies is associated with inflammatory bowel disease. $\mathcal{F}$ Allergy Clin Immunol 1990; 86: 202-10.

7 Duerr R H, Targan S R, Landers J, et al. Neutrophil cytoplasmic antibodies: a link between primary sclerosing cholangitis and ulcerative colitis. Gastroenterology 1991; 100: 1385-91.

8 Nässberger L, Sjöholm A G, Jonsson H, Sturfelt G, Akesson A. Autoantibodies against neutrophil cytoplasm components in systemic lupus erythematosus and in hydralazine-induced lupus. Clin Exp Immunol 1990; 81: 380-3.

9 Van der Woude F J, Rasmussen N, Lobatto S, et al. Autoantibodies against neutrophils and monocytes: tool for diagnosis and marker of disease activity in Wegener $s$ for diagnosis and marker of disease activity

10 Falk R J, Jennette J C. Anti-neutrophil cytoplasmic autoantibodies with specificity for myeloperoxidase in patients with systemic vasculitis and idiopathic necrotizing and crescentic glomerulonephritis. $N$ Engl f Med 1988; 318: 1651-7. 